\title{
EchoGéo
}

$33 \mid 2015$

Activisme, participation, contestation : la place des habitants dans les processus de patrimonialisation en périphéries urbaines

\section{Les nouvelles causes du patrimoine. L'exemple du Musée Urbain Tony Garnier à Lyon}

\section{Alain Chenevez}

\section{OpenEdition}

\section{Journals}

\section{Electronic version}

URL: https://journals.openedition.org/echogeo/14346

DOI: 10.4000/echogeo.14346

ISSN: 1963-1197

Publisher

Pôle de recherche pour l'organisation et la diffusion de l'information géographique (CNRS UMR 8586)

\section{Electronic reference}

Alain Chenevez, "Les nouvelles causes du patrimoine. L'exemple du Musée Urbain Tony Garnier à Lyon ", EchoGéo [Online], 33 | 2015, Online since 30 September 2015, connection on 21 September 2021. URL: http://journals.openedition.org/echogeo/14346 ; DOI: https://doi.org/10.4000/echogeo.14346

This text was automatically generated on 21 September 2021.

EchoGéo est mis à disposition selon les termes de la licence Creative Commons Attribution - Pas d'Utilisation Commerciale - Pas de Modification 4.0 International (CC BY-NC-ND) 


\title{
Les nouvelles causes du patrimoine. L'exemple du Musée Urbain Tony Garnier à Lyon
}

\author{
Alain Chenevez
}

\section{Les champs du patrimoine}

1 Le patrimoine est un pur produit de l'histoire. Mais contre le sens commun qui le présente comme un bien qui s'imposerait à nous, l'observation scientifique montre que c'est au contraire un artefact créé de toutes pièces (Heinich, 2009). Son objet est éclectique, protéiforme et concerne des éléments et avoirs, aussi bien matériels qu'immatériels (Bortolotto, 2011). Pour résister à la finitude, son objectif est de prendre soin de ce qui nous entoure, de s'intéresser, de désigner, de préserver, de mettre en valeur, de conserver, le cas échéant de transmettre des valeurs d'héritage. Une de ses fonctions initiales est d'organiser et de signifier le principe de la succession des générations, parfois en se cristallisant sous des formes institutionnelles et publiques (Legendre, 2004). Mais force est de constater que rien ne s'impose, telle une évidence, en matière de patrimoine. Pour qu'il y ait conscience de l'héritage, il faut un travail politique de désignation mais aussi d'appropriation, sinon l'oubli prime. Le passé est certes structurant, mais c'est bien du présent que nous inventons un legs et sa signification (Davallon, 2000). Il faut donc s'intéresser aux conditions sociales dont il est le produit et comment il se réinvente à chaque génération. Le patrimoine est toujours un processus de tri et de qualification du présent de ce qui doit perdurer et/ou être mis en avant pour séparer l'important du futile, l'insignifiant de ce qui compte. C'est le cas échéant une convention qui encadre l'appartenance, la dépendance et la possession, l'affiliation et les hiérarchies sociales. Sans présager davantage sur ce point ici, nous assistons depuis plusieurs décennies à une extension importante de ce qui fait patrimoine, en tout cas à une prolifération de ce qui est désigné comme tel. Le mot est de plus en plus utilisé par le milieu associatif et marchand, pour désigner des objets, des sites, des pratiques, des espaces (Saez, Glévarec, 2002). Loin de se réduire au 
concept de Monuments Historiques ou d'héritage domestique, il est même devenu un Janus conceptuel. Faute d'expliquer le phénomène d'extension dont elle est en partie responsable, la recherche anthropologique le comprend plutôt par un processus pragmatique et sensible pour définir ce qui nous attache (Fabre, 2011), ce à quoi nous portons de l'intérêt, ce à quoi nous tenons, et de quoi nous dépendons (Hache, 2011; Tornatore, 2015). C'est une valeur sociale et symbolique qui évolue selon les époques et qui a un rapport qualifiant avec le temps et l'espace, mais aussi avec les mots et les choses. Il est produit par des logiques d'acteurs qui le signifient en le désignant, qui le mettent en relation avec des réseaux de consécration. En somme, c'est un choix sur ce qui doit être désigné comme symbole et bien commun après une évaluation collective.

\section{Pour une géographie critique du patrimoine}

2 De manière singulière, les recherches en géographie sur la dimension spatiale de ce qui est désigné aujourd'hui comme patrimoine dans l'espace urbain soulignent que ce processus est inégalitaire. Nous ne tenons pas à tout de la même manière et le soin que l'on porte aux espaces n'est pas égal, loin s'en faut. Les quartiers socialement valorisés ou centraux en Europe font souvent l'objet de préservation, de mise en valeur par l'inscription aux Monuments Historiques ou à des labels nationaux ou internationaux par exemple avec l'Unesco, quand d'autres sont détruits ou ne font pas l'objet de distinction ou de singularisation positive (Veschambre, 2008). L'arrivée du patrimoine et sa désignation dans un espace préfigurent dans ce sens la gentrification, c'est-à-dire la transformation sociologique et la venue de nouvelles catégories sociales par l'esthétisation et la valorisation foncière qui en découle (Ter Minassian, 2013). La désignation patrimoniale provoque un intérêt renouvelé sur l'espace ainsi désigné et une hausse des loyers le cas échéant. Plus encore, la rhétorique de la préservation des espaces permet à certains groupes sociaux de faire reconnaître leur cadre de vie, leur quartier, leur trace, leur espace comme ayant valeur d'héritage aux yeux de tous, soulignant une capacité de certains acteurs à universaliser des propriétés singulières et locales (Melé, 2013). Autrement dit le patrimoine lié aux espaces urbains serait en partie un outil de distinction masquant derrière le bien commun des enjeux de qualifications spatiales et sociales et d'appropriations des crédits alloués.

\section{Les nouvelles « causes » du patrimoine}

3 Sans nier l'intérêt de ces analyses, nous souhaiterions prendre nos distances avec cette perspective critique qui ne permet pas de rendre compte des prétentions à la justice patrimoniale de certains acteurs ces dernières décennies. Inspirés par les travaux de Luc Boltanski et de Laurent Thévenot et leurs économies de la grandeur, nous souhaiterions montrer que le patrimoine est aussi un travail interprétatif et argumentatif qui s'apparente à la fabrication d'une "cause " (Boltanski, Thévenot, 1991). De nouveaux arguments issus en partie du relativisme culturel mais aussi des sciences humaines et sociales permettent de légitimer de nouveaux espaces et de désigner des artefacts originaux. Les logements sociaux par exemple ne connaissaient pas ou peu de désignation patrimoniale. Les cités d'habitat social sont peu concernées par le patrimoine, souvent considérées comme des lieux d'exil qui concentrent des catégories estimées comme dangereuses (Dubet, Lapeyronnie, 1999). Cela étant, grâce aux 
arguments de l'anthropologie mais aussi de l'histoire populaire et de la sociologie, des opérations de dévoilement patrimonial émergent sur des territoires souvent peu considérés. Nous étayerons notre propos en soulignant le nécessaire travail de construction d'une « cause » patrimoniale, nous nous pencherons sur une mobilisation inaccoutumée d'un quartier d'habitat social. Nous décrirons l'expérience originale du Musée Urbain Tony Garnier à Lyon qui s'est produite dans un quartier d'habitats populaires en soulignant ses réussites et ses écueils. Ce projet possède une certaine antériorité car c'est un processus qui a débuté au cours des années 1980. Il nous interroge particulièrement sur la capacité des populations locales à s'impliquer dans un processus de (re)qualification et de (re)valorisation.

La méthodologie utilisée provient de mes connaissances, de mon parcours, d'archives privées, d'entretiens et de mes observations participantes en tant que sociologue mais aussi directeur de cette institution patrimoniale naissante présente dans un quartier d'habitat social au cours des années 2000. Revenons sur une histoire de singularisation d'une cité habitée.

Illustration 1 - Vue d'une rue intérieure de la Cité Tony Garnier, Quartier des États-Unis, Lyon 8 e

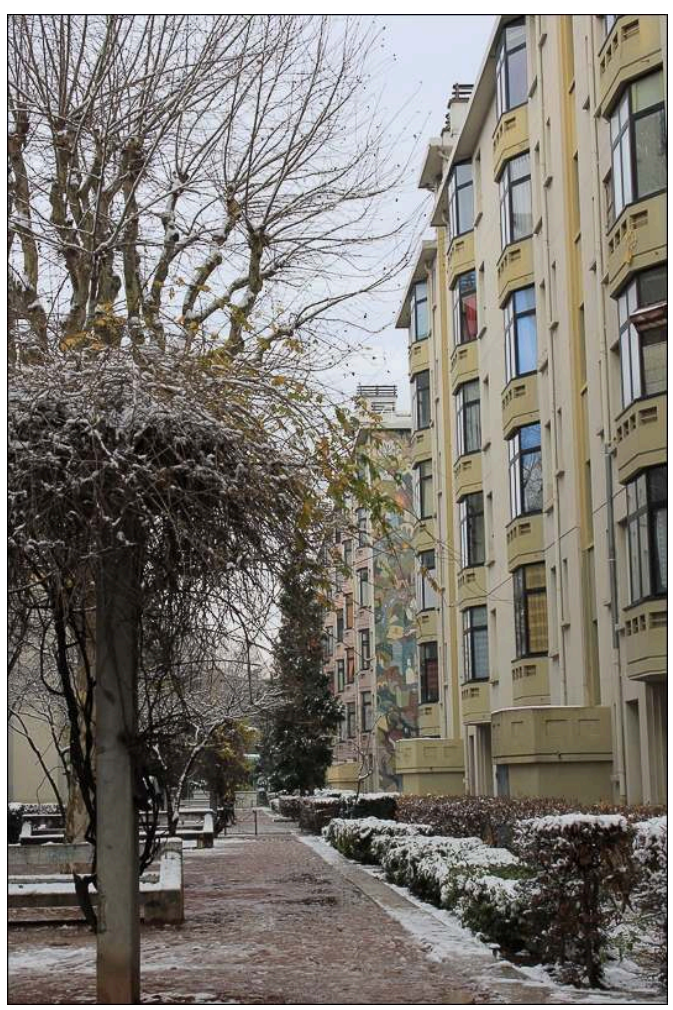

Auteur A. Chenevez. 


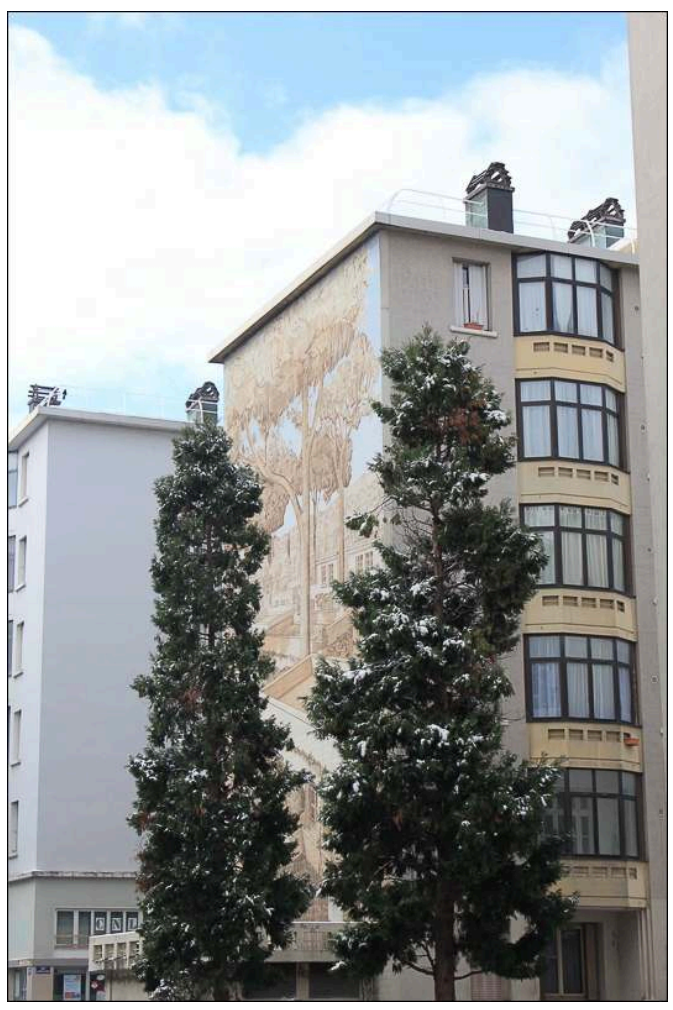

Auteur : A. Chenevez.

\section{L'exemple de la Cité Tony Garnier à Lyon : une histoire singulière}

À l'est de la ville de Lyon, à quelques centaines de mètres de la commune de Vénissieux, un programme d'environ 1670 logements est construit, entre 1924 et 1935, par l'architecte Tony Garnier. Le vaste plan d'habitats collectifs à destination principalement des ouvriers est alors l'un des plus grands d'Europe. Tony Garnier préconise pour la zone d'habitations bon marché une architecture pratique avec un programme de bâtiments réalisés en îlots. L'utilisation de techniques industrielles de construction en ciment armé et mâchefer permet de réduire les coûts de fabrication. L'objectif est de loger les classes laborieuses dans des appartements agencés et accommodés au confort moderne. Tony Garnier ne réalise cependant pas tout à fait le programme initial. Aucun bâtiment administratif et d'éducation n'est alors réalisé dans le quartier des Etats-Unis. L'architecte doit en plus accepter d'ajouter un ou deux étages aux immeubles imaginés au départ pour permettre une plus grande densité (Roz, 1980). Cela étant, l'ensemble est inauguré en 1935 en grande pompe. Ce nouveau quartier est considéré comme une avancée républicaine importante par les édiles lyonnais ${ }^{1}$. Les locataires sont choisis après enquête de moralité, par cooptation ou parfois même par clientélisme affiché comme le soulignent les recherches de Claire Berthet. Ainsi, la plupart des entrants dans ces nouveaux immeubles sont des ouvriers et employés dont la stabilité professionnelle est avérée. Presque $10 \%$ appartiennent même aux classes moyennes et supérieures: médecins, enseignants, ingénieurs (Berthet, 2013). 
6 Il faut attendre les années 1960-1970 pour qu'une nouvelle urbanisation se réalise avec l'érection au cours de cette période de nouveaux logements sociaux sur plus de deux kilomètres entourant la Cité. Ces nouvelles constructions n'ont aucun lien avec le projet de Tony Garnier. Mais elles contribuent à l'intégration de l'ancienne cité dans le tissu urbain, aussi à sa normalisation et dé-singularisation. Le quartier des États-Unis qui incarnait le progrès social et républicain se dégrade peu à peu, tout comme l'ensemble des quartiers de logements sociaux au cours des années 1970-1980 (Masclet, 2005).

\section{La carrière patrimoniale de la Cité Tony Garnier}

$7 \mathrm{Au}$ milieu des années 1980, cinquante ans après sa construction, l'ensemble architectural de Tony Garnier frappe par sa décrépitude. Des locataires et le comité impulsent et appuient une opération de réhabilitation, à partir des années 1985, conduite par l'Office public communautaire HLM de Lyon, propriétaire de l'ensemble des bâtiments. Invités par les résidents, de nouveaux alliés s'intègrent à un projet de rénovation qui au démarrage était très classique, mais qui s'avère difficile à mettre en œuvre. Les artistes de la Cité création et leur Centre de recherche "Esthétique et Cités ", alors spécialisés sur les interventions en milieu urbain et formés pour certains à la sociologie, proposent de réaliser sur les murs pignons des bâtiments des peintures murales (Forêt, 1992). L'idée est, dès le départ, de représenter les dessins de l'architecte et de réaliser un musée en plein air ouvert à tous pour re-distinguer, en quelque sorte, la cité dégradée de Tony Garnier et apporter de la dignité à un quartier populaire. Le but est de répondre à une sollicitation des habitants. Plus encore, l'espoir est d'accélérer ainsi le processus de réhabilitation, de le rendre obligatoire auprès de l'office HLM et des collectivités locales. Mais il ambitionne aussi de valoriser un concept d'intervention esthétique et colorée en milieu urbain que les artistes tentent alors d'imposer dans le paysage lyonnais. 


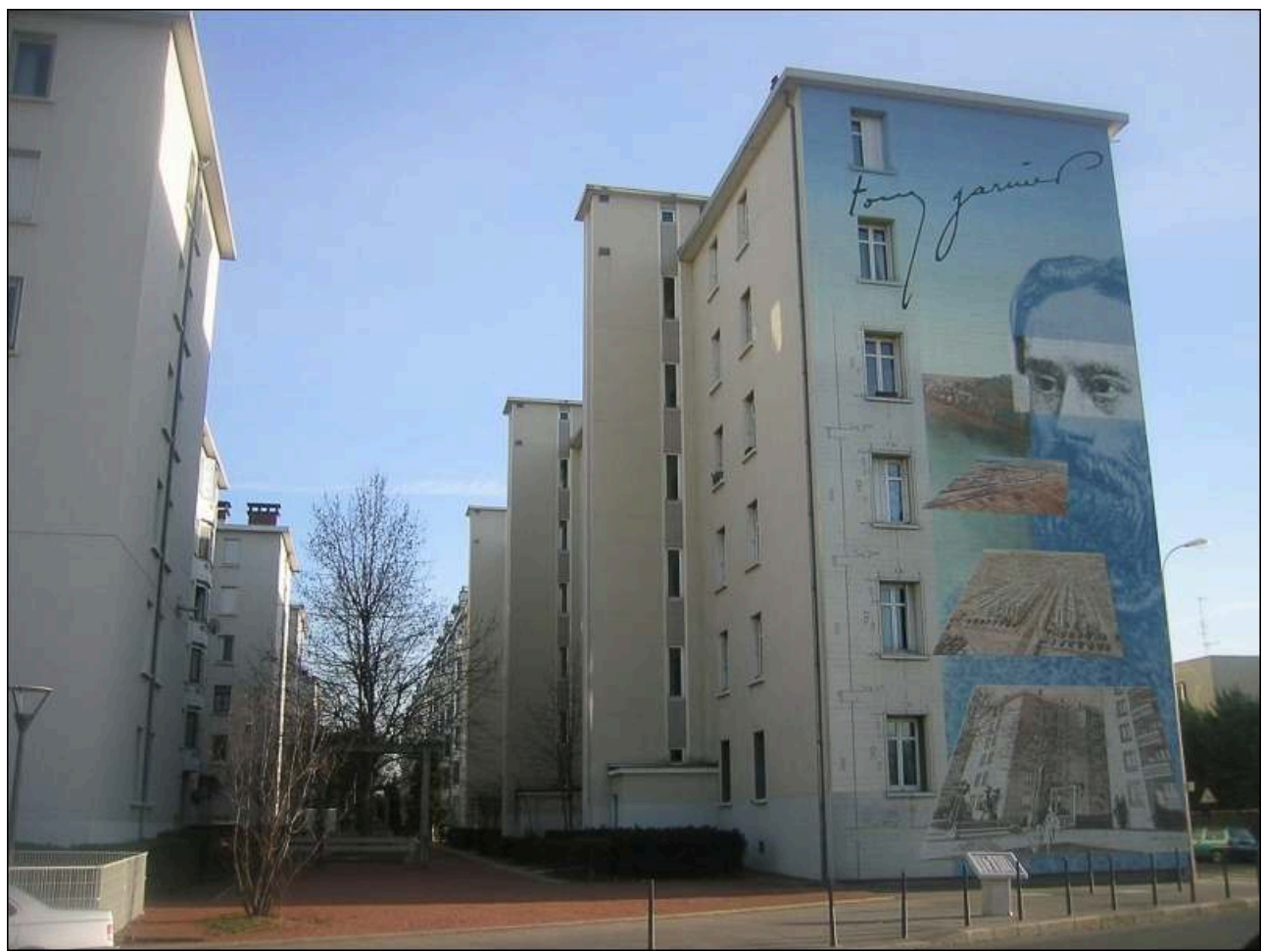

Auteur : A. Chenevez; @ Musée Urbain Tony Garnier.Concept et réalisation de CitéCréation.

\section{Une action collective originale hors des sentiers de l'administration culturelle}

8 Ce programme d'esthétique urbaine ne sera jamais soutenu par le Ministère de la culture. Ce dernier ne souhaite pas la réalisation de ce projet de fresques monumentales dans un quartier d'habitat social. La Direction régionale des affaires culturelles ne considère pas ce programme comme un projet artistique, tout au plus est-il perçu comme de l'accompagnement socio-culturel. Les zones périphériques et les artistes de Cité création ne sont pas la préoccupation de cette administration et l'architecte Tony Garnier suscite de même peu l'intérêt du monde muséal ${ }^{2}$.

Le soutien viendra du Préfet du Rhône, Gilbert Carrère, et des agents de la Politique de la ville, nouvellement nommés dans le cadre du projet Banlieue 89. La Direction Départementale de l'Équipement et des fonds de rénovation urbaine appuient financièrement le projet de peintures murales et de Musée urbain en plein air ${ }^{3}$ (Carrère, 2012).

10 En effet, l'aspect patrimonial est un obstacle au financement et ne permet pas de lever les fonds nécessaires. Sans légitimité institutionnelle, c'est par le biais d'une rénovation technique, l'amélioration thermique des bâtiments, que ce projet esthétique se concrétise et ce malgré les réserves importantes de l'administration culturelle.

«Cela ne rentrait pas vraiment dans les cases... Ce n'est pas un musée de toute façon, en plus il y avait un problème au niveau de la forme artistique... des fresques. C'était au mieux socio.... nous ne pouvions pas soutenir cela, et

en plus les peintures murales c'est très moyen il faut bien reconnaître, nous 
n'étions pas sur cette orientation. Tony Garnier nous intéressait, mais pas comme cela... » (Cf. entretien avec un ancien fonctionnaire de la Direction des Affaires culturelles). légitimer le projet, notamment grâce à l'implication des populations locales ${ }^{4}$. Ce projet consonne avec les priorités d'une politique publique dont le but est de revaloriser les zones urbaines en difficulté mais, autant que possible, en relation étroite avec les principaux intéressés (Donzelot, 2003). Les agents chargés de mettre en place cette politique à Lyon ne sont alors pas très nombreux. Ils sont tout au plus deux ou trois, mais ils contribuent fortement à l'émergence du projet en soutenant le programme et en lui donnant une légitimité institutionnelle.

Depuis la loi du 31 décembre 1982, les arrondissements sont transformés en structures déléguées à l'échelon local. C'est une opportunité politique pour un maire de «centre droit ", Robert Batailly, de répondre à une demande locale émanant d'un quartier populaire alors en difficulté matérielle. Il milite activement auprès des pouvoirs centraux de la commune pour susciter l'intérêt d'un financement plus important (Cf. entretien Catherine Panassier, 2009). Tous finiront par convaincre le président de l'office public d'HLM. Celui-ci, contraint de répondre favorablement à la pression des résidents, mais aussi de la mairie, aux artistes et au préfet finit par impliquer son équipe en tant que maître d'œuvre et d'ouvrage sur l'opération. L'État par l'intermédiaire du Secrétariat permanent du Plan Urbain contribue également. Il finance, au tout début du projet, des études de sociologie pour mieux comprendre les enjeux dans une perspective d'aménagement $d u$ territoire avec une dimension esthétique. La recherche scientifique renforce le bien fondé d'un processus qui repose principalement sur la participation des populations locales et le lien social induit par les premières peintures et la naissance de l'association Perspectives Tony Garnier ${ }^{5}$. 


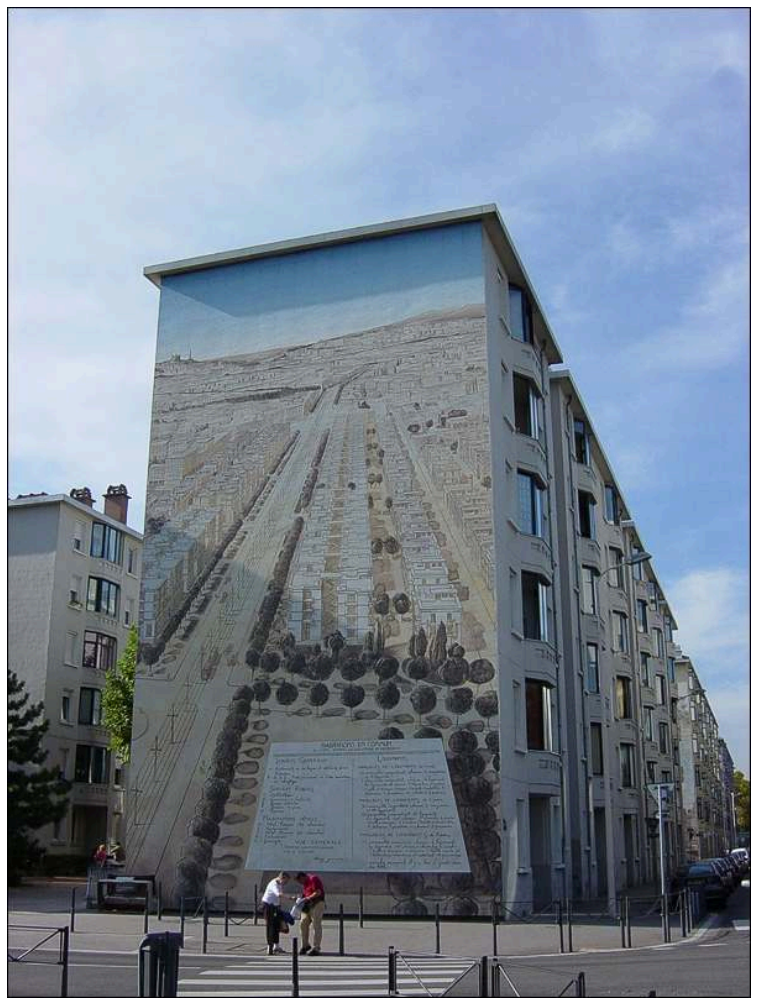

Source : Concept et réalisation de CitéCréation.

\section{La cause patrimoniale liée à l'implication des habitants}

14 Autrement dit et sans être ici exhaustif, de nombreuses personnalités, institutionnelles et/ou associatives, contribuent par leurs actions à l'émergence d'une convention et d'une cause patrimoniale singulière autour de l'implication habitante, au-delà de l'aspect artistique.

«Nous ne sommes pas des artistes, nous sommes des artisans en peinture » nous dira un des artistes de Cité création.

«On nous amenait sur le terrain de la discussion pour nous faire entrer dans des débats sans fin, mais nous on sort de la dispute, il y avait urgence à réhabiliter, donc on dit ce n'est pas de l'art ce que l'on fait, on fait de l'esthétique urbaine. D'abord, on n'appartenait pas à ce monde-là, on avait quitté le monde de la culture pour rentrer dans le monde du bâtiment. On ne voulait pas ferrailler avec eux mais faire ailleurs ! On ne pouvait rien peindre sur du Tony Garnier, à moins de peindre du Tony Garnier » (Entretien avec Gilbert Coudène, septembre 2014).

16 En ce sens, la singularité de ce projet esthétique est de se définir dans la figure emblématique de Tony Garnier, mais plus encore de se justifier dans une valeur d'usage prenant en compte la dimension citoyenne et de critiquer les inégalités en matière de culture. C'est un processus collectif dont une des caractéristiques est d'éviter la « dispute » liée à la dimension artistique en s'écartant de la culture institutionnelle et 
en soulignant l'urgence d'une situation. Ce détour qualifie la valeur à apporter à ce projet, le fait exister dans l'implication des résidents et renforce ainsi son efficacité par l'évacuation de la controverse culturelle.

Cela étant dit, même si les populations locales sont au cœur du récit et de l'argumentaire, elles n'imposent pas seules l'intérêt autour de Tony Garnier et des peintures. Même si leur intervention est capitale pour la réussite du projet, les habitants trouvent des alliés extérieurs au quartier, sans quoi l'intention risque de rester lettre morte. L'artefact produit est le résultat d'une configuration d'agents et d'acteurs qui fabriquent un bien commun sui generis, chacun dans un système de dispositions et d'interprétation singulières, avec des formes d'ajustements et de négociations. Sans cobelligérants sur des postes clés que ce soit au niveau de la Ville de Lyon, de l'Office HLM ou encore de l'État, le projet n'aurait pas pu se concrétiser. Les personnes et les institutions ayant contribué dans un premier temps, au cours des années 1980-1990, à la naissance de cet objet culturel non identifié, collaborent chacune à leur manière, à la cause sans avoir ni la même histoire, ni les mêmes intérêts et les mêmes visions des choses (Becker, 1988). Comme dans le générique d'un film, il y a des rôles principaux et secondaires. Tony Garnier, les habitants et les artistes de Cité création sont les acteurs centraux. En référence aux travaux d'Howard Becker, mais aussi de Luc Boltanski et Laurent Thévenot, nous avancerons l'hypothèse que c'est la forme sociale de tous qui est fondamentale à la réussite d'une cause patrimoniale (Boltanski, Thevenot, 1991) en dehors des institutions culturelles. La configuration sociale se matérialise par des micro-stratégies, des alliances improbables, des interactions et des controverses, des luttes et des conflits, mais surtout un consensus minimum sur le but à atteindre. La convention mise en avant pour imposer une plusvalue patrimoniale, c'est l'implication des résidents d'un quartier populaire dans la réhabilitation esthétique de leur cadre de vie, leur volonté de participation aux affaires de la Cité. Cette dimension participative issue de la socio-anthropologie est vécue comme un bon droit de justice pour construire une "grandeur ", un bien commun, une morale. Plus encore, la culture légitime et institutionnelle sert ici de repoussoir. La sociologie de la culture est alors utilisée pour souligner le caractère inégal de l'intérêt culturel et patrimonial. « ...qui va au musée? Tu connais comme moi, donc on s'est dit, on va amener le musée à eux tu vois " me dira lors d'un entretien l'un des fondateurs pour légitimer son action.

Cet accord sur l'implication permet une cohésion militante vécue comme fondée et juste. En d'autres termes, nous sommes devant un système original de relations efficaces pour faire émerger une valeur patrimoniale portée par des conventions d'appropriations citoyennes, en puisant dans la sociologie critique certains arguments. Mais plus encore, ces interactions se pérennisent dans des réseaux supports de l'appartenance commune et de la cristallisation d 'une " grandeur " patrimoniale. Tony Garnier n'était pas suffisamment reconnu et personne ne disposait alors des ressources nécessaires pour le convoquer seul. L'herméneutique de son œuvre architecturale restait à écrire. Il fallait déplacer les arguments, faire un détour pour éviter la controverse artistique et au bout du compte faire « œuvre d'art participative» dans l'imaginaire collectif. 
Illustrations $5 a$ et $5 b$ - Immeubles de la Cité Tony Garnier avec une fresque du quartier de la gare de la Cité industrielle : vue perspective

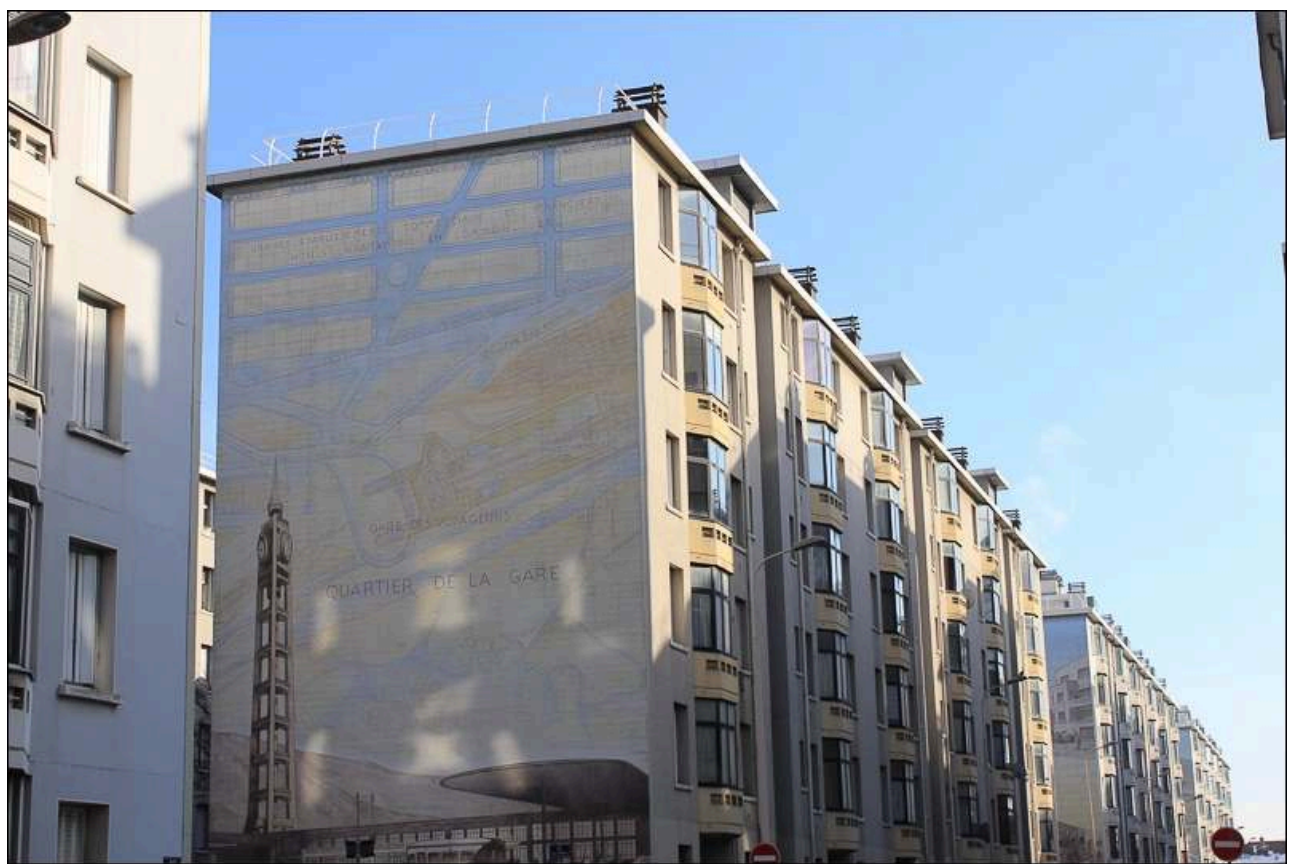

Auteur : A. Chenevez ; @Musée Urbain Tony Garnier.Concept et réalisation de CitéCréation.

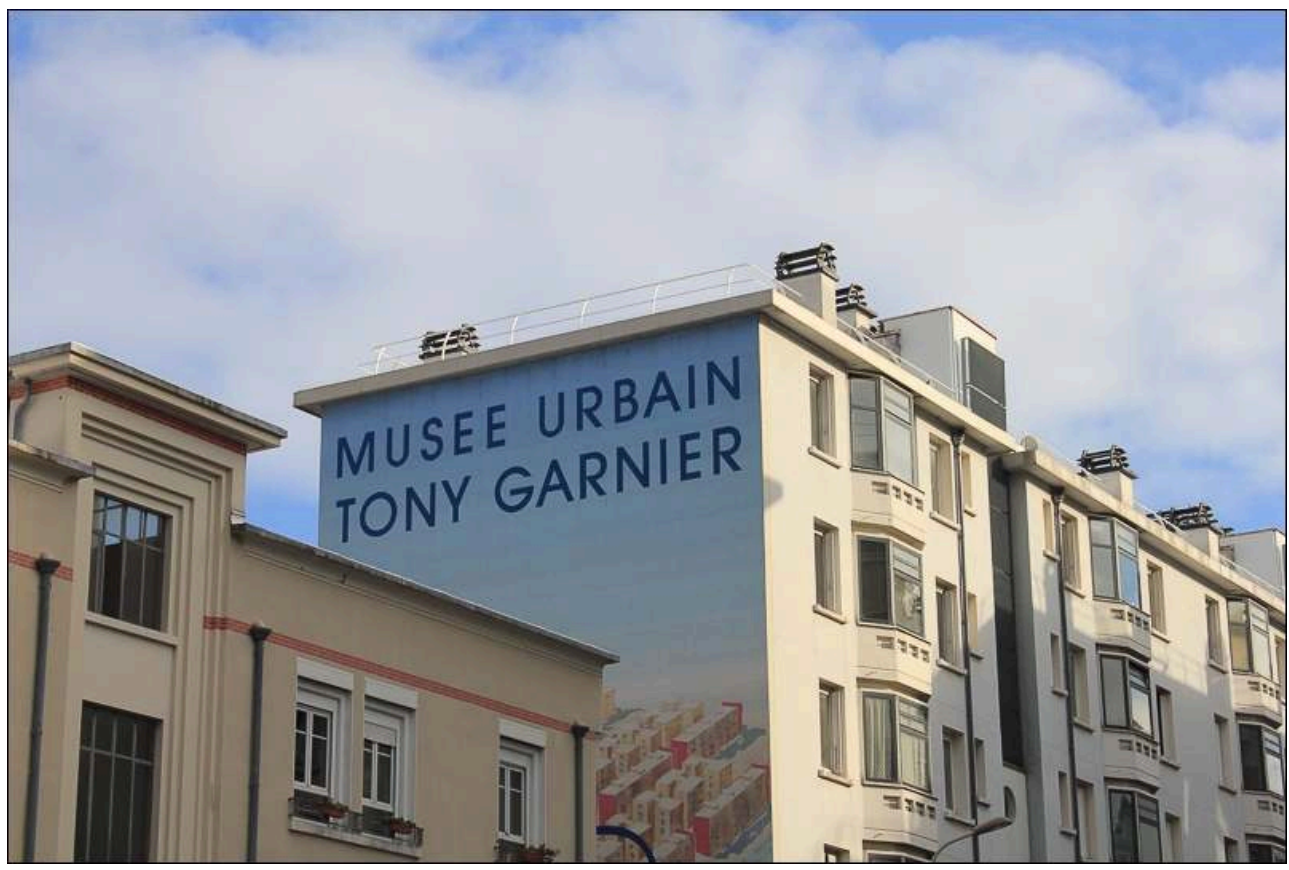

Auteur : A. Chenevez; @Musée Urbain Tony Garnier. Concept et réalisation de CitéCréation.

\section{Le cadre militant des habitants}

Les résidents de la cité qui s'investissent sont pour la plupart des anciens ouvriers assez âgés, souvent des femmes très proches du Parti communiste et/ou investis dans les luttes syndicales ou associatives et qui trouvent dans l'énergie d'un plus jeune, Eddie Gilles Di Pierno ${ }^{6}$, un levier pour se requalifier symboliquement et se distinguer. Souvent 
issus de l'immigration italienne et vivant pour la plupart dans les bâtiments des années 1930, les militants se sentent identiques à des propriétaires et vivent mal la dégradation symbolique que connaissent les logements sociaux au début des années 1980 (Catherine Foret, 1993). Ils ne veulent pas être associés aux nouveaux quartiers d'habitats sociaux et aux nouvelles catégories populaires qui semblent être moins soucieuses de leur environnement. La valorisation initiale, le sentiment d'incarner une modernité républicaine, ajoutés à la lente dégradation du statut matériel des bâtiments de Tony Garnier semblent, pour Catherine Foret, des éléments essentiels pour comprendre les formes de cette action collective qui mobilise parfois jusqu'à 300-400 personnes sur le quartier. Nous avancerons que cette sociologue observe et donne aussi des arguments critiques pour légitimer l'action qu'elle analyse. Alors que ce projet connaît des hauts et des bas, la chaîne de collaboration d'acteurs s'enrichit d'une reconnaissance symbolique internationale.

\section{La reconnaissance internationale de l'Unesco : le label de la Décennie mondiale du développement}

Nous sommes en 1991 et l'Unesco instruit un dossier de labellisation du projet du Musée Urbain Tony Garnier au titre de la Décennie mondiale du développement ${ }^{7}$. Le Musée Urbain semble correspondre à un programme non permanent de l'Unesco qui vise, entre 1988 et 1997, à convaincre décideurs politiques, acteurs sociaux locaux et nationaux d'intégrer de la diversité et du pluralisme dans l'ensemble des politiques culturelles, via notamment des partenariats public/privé, ce qui était tout à fait le cas ici ${ }^{8}$. Cette reconnaissance internationale permet de relancer un projet en difficulté en 1991. En effet, le maire de Lyon à ce moment là, Michel Noir, souhaite faire réaliser des fresques monumentales dans d'autres quartiers pour réaliser un parcours sur l'architecte ${ }^{9}$. Cette perspective aurait très vraisemblablement mis à mal l'implantation du Musée urbain sur le quartier des États-Unis. La labellisation au titre de la Décennie mondiale par l'Unesco arrive à point nommé et relance le projet. Elle permet en grande partie de redonner de la légitimité, de re-fonder l'accord par la reconnaissance extérieure et institutionnelle. Six nouvelles peintures murales sont réalisées, entre juin 1993 et novembre 1995. La presse locale souligne alors que le Musée Urbain est entré dans une phase internationale ${ }^{10}$. 


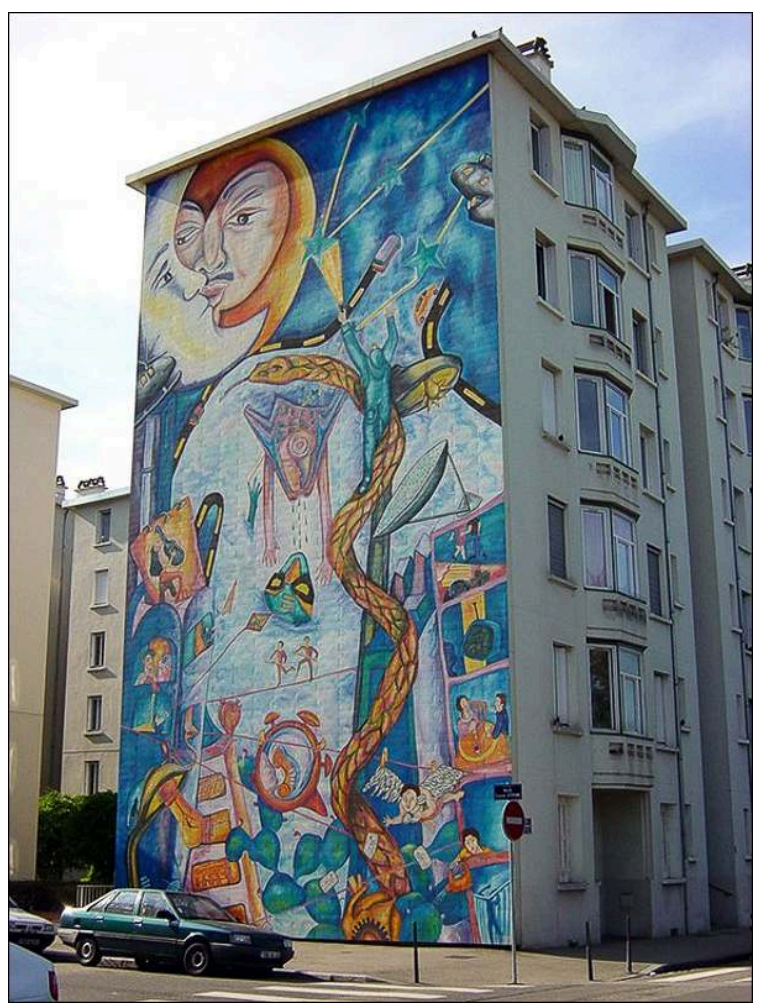

Source : @ Musée Urbain Tony Garnier. Concept et réalisation de CitéCréation,

\section{Une fabrique patrimoniale contributive}

Cette première phase de fabrication que nous pourrions appeler la fabrique argumentative, s'organise autour d'acteurs qui militent chacun à leur manière et produisent par leur influence, de la légitimité et une valeur d'usage esthétique pour ce qui relevait jusqu'alors de l'ordinaire. Il fabrique une cause patrimoniale autour de valeurs portées par la politique de la ville, dans l'appropriation contextuelle des espaces par des résidents et nourrie de sociologie critique mais aussi d'anthropologie et de diversité avec le cas de l'Unesco. Il s'agit ainsi de rendre le site agréable, de renforcer l'adhésion des populations locales, mais aussi vis à vis de l'extérieur, de modifier par l'impact esthétique l'image du quartier dans la ville, le réinstaller dans une modernité, contrer le déclin et l'inscrire dans un nouvel imaginaire. Les principaux arguments qui se développent dans l'action tentent de s'inscrire dans un rapport de justice par la remise en cause du bien-fondé des hiérarchies culturelles. Il y a là une exigence morale qui se construit dans la critique, dans la confrontation mais aussi l'évitement, dans l'argumentation sans cesse renouvelée, au nom de principes présentés comme d'intérêt général et juste. Vrai ou faux, populiste ou misérabiliste, ce n'est pas ici la question. L'idée était de dépasser le constat de la socio-anthropologie d'une domination insurmontable des institutions et objets patrimoniaux consacrés pour s'inscrire dans une logique d'émancipation et d'imposition de nouvelles normes de légitimité patrimoniale plus citoyennes (Chenevez, 2005). Cette première phase liée à la fabrication des peintures murales dure une douzaine d'années et s'achève en 2001, avec la réalisation d'une dernière peinture sur un bâtiment appartenant à la SACVL 
(Société Anonyme de Construction de la Ville de Lyon). Cette opération de réhabilitation s'est donc concrétisée par la fabrication de 25 peintures murales de 230 mètres carrés chacune entre 1989 et 2001 et également par la naissance d'une structure muséale, par l'intermédiaire d'une association, dont l'objectif est de promouvoir cette expérience. Le Musée Urbain Tony Garnier, proche du centre d'interprétation ou de l'écomusée par les principes d'implication des populations locales (cf. Hugues De Varine, 2005) voit se succéder des équipes de bénévoles et quelques salariés qui organisent la mise en place d'éléments de médiation et d'excursion touristique jusqu'à aujourd'hui (Cf. www.museeurbaintonygarnier.com). De même, un appartement-musée est entièrement meublé années 30 et scénographié par les habitants pour y organiser des visites.

\section{La professionnalisation de la structure et l'éviction des habitants}

Pour prolonger ce programme lié aux peintures murales, l'objectif est de déployer l'association et faire vivre une structure qui, selon le Conseil d'administration d'alors, doit se professionnaliser. Ajoutons aussi le besoin de trouver de nouvelles formes de légitimité pour survivre, se " prémunir » et pérenniser l'expérience. Sans cela, le déclin semble inévitable (Cf. Compte rendu Conseil d'administration du 6 décembre 2000 et rapport d'activités daté du $1^{\mathrm{er}}$ semestre 2001, archives privées).

Le Conseil d'administration de l'association est alors composé d'un tiers d'habitants, d'un tiers d'élus et d'un tiers de personnalités extérieures. Ils conviennent de poursuivre l'aventure autrement, les murs pignons étant désormais tous peints, l'idée s'impose de les valoriser. Un sociologue est recruté pour imaginer la rédaction d'un projet d'établissement puis d'un projet scientifique qui doit donner de l'épaisseur théorique. À nouveau, les arguments critiques de la sociologie, le caractère inique des politiques culturelles et inégal de la production patrimoniale sont mis en avant pour faire en quelque sorte justice. (Cf. projet d'établissement 2004-2008 rédigé par Sébastien Cavalier et Alain Chenevez, archives privées). Le principe est de diversifier et d'enrichir l'offre par la mise en place d'une politique d'expositions temporaires. Il s'agit aussi de développer, de diffuser et de promouvoir les études sur Tony Garnier, par la mise en place d'un centre de ressources et d'un fonds Tony Garnier. Puis à terme de créer un centre d'interprétation du patrimoine sur la problématique Art et urbanisme, culture et renouvellement urbain. Bref, l'orientation choisie est de se normaliser pour entrer dans une nouvelle perspective plus muséographique. L'exégèse sur Tony Garnier s'est étoffée et peut désormais être un outil utile pour légitimer le développement de l'institution. La finalisation du récolement des fonds documentaires, le lancement d'un programme de photographie et de numérisation, la mise en place d'éléments de médiations, de visites audioguidées, l'esthétisation des espaces d'accueil et d'expositions, les recherches sur Tony Garnier, la valorisation des publications existantes, la mise en place d'un pôle muséal associé avec le Musée Gadagne, tout devait convaincre des institutions publiques et privées du bien-fondé de cette institution atypique. L'autofinancement insuffisant nécessite la recherche de mécénat pour les actions et les évènements. Il faut avant tout répondre à un cadre normatif pour obtenir les moyens de la survie financière. Seulement, l'administration culturelle des collectivités territoriales est moins intéressée par la participation des habitants que par 
la qualité du projet de développement culturel et des programmations. Malgré les difficultés et même si la structure est encore aujourd'hui fragile, la stratégie porte en partie ses fruits. La ville de Lyon double son budget en 2006 et finance la structure sur des crédits " culture ». Il en est de même de la Région Rhône-Alpes, ce qui permet de boucler le financement d'une salle d'exposition de 300 mètres carrés et l'embauche d'une petite équipe de 5 personnes. Même l'État par l'intermédiaire de la Direction des affaires culturelles finit par octroyer une subvention pour des actions liées à l'architecture à partir de 2006.

Ainsi la cause patrimoniale ne passe plus prioritairement par l'implication locale mais par une orientation plus institutionnelle, portée par l'exégèse de l'architecte Tony Garnier. Le processus est quasiment inéluctable. Malgré des arguments très liés au participatif et au lien social induit par ce type de projet, le développement entre ici en contradiction avec les conventions initiales et provoque une coupure durable de l'association du Musée Urbain Tony Garnier avec son environnement immatériel immédiat. Les résidents de la Cité et plus encore les acteurs en quelque sorte historiques du projet sont irrémédiablement écartés du Conseil d'administration au profit d'une expertise extérieure et d'un marquage politique par les élus locaux. Dans cette optique, l'énergie donnée par les salariés de l'association se concentre sur le projet scientifique autour de Tony Garnier, voire sur l'animation touristique et non sur des actions participatives. Exit les habitants et bienvenue aux architectes, aux universitaires, aux artistes d'art contemporain aux conservateurs, mais aussi aux médiateurs touristiques qui apportent la valeur symbolique nécessaire à l'obtention des financements. Lentement les militants locaux ne sont plus les porteurs du projet et sont perçus comme des freins au développement. Comme s'il fallait s'oublier pour se réinventer, en quelque sorte. Le Musée Urbain Tony Garnier doit s'inscrire dans un nouveau système de relations et de légitimité. Dans cette optique, il doit être porté par ceux capables de se faire entendre, c'est-à-dire les classes moyennes " politisées », des universitaires, des journalistes, des élus; reconstituant une forme d'élitisme et d'évacuation du populaire. 
Illustration 7 - Vue de l'espace d'accueil et la salle d'exposition du Musée Urbain Tony Garnier inaugurés en 2006

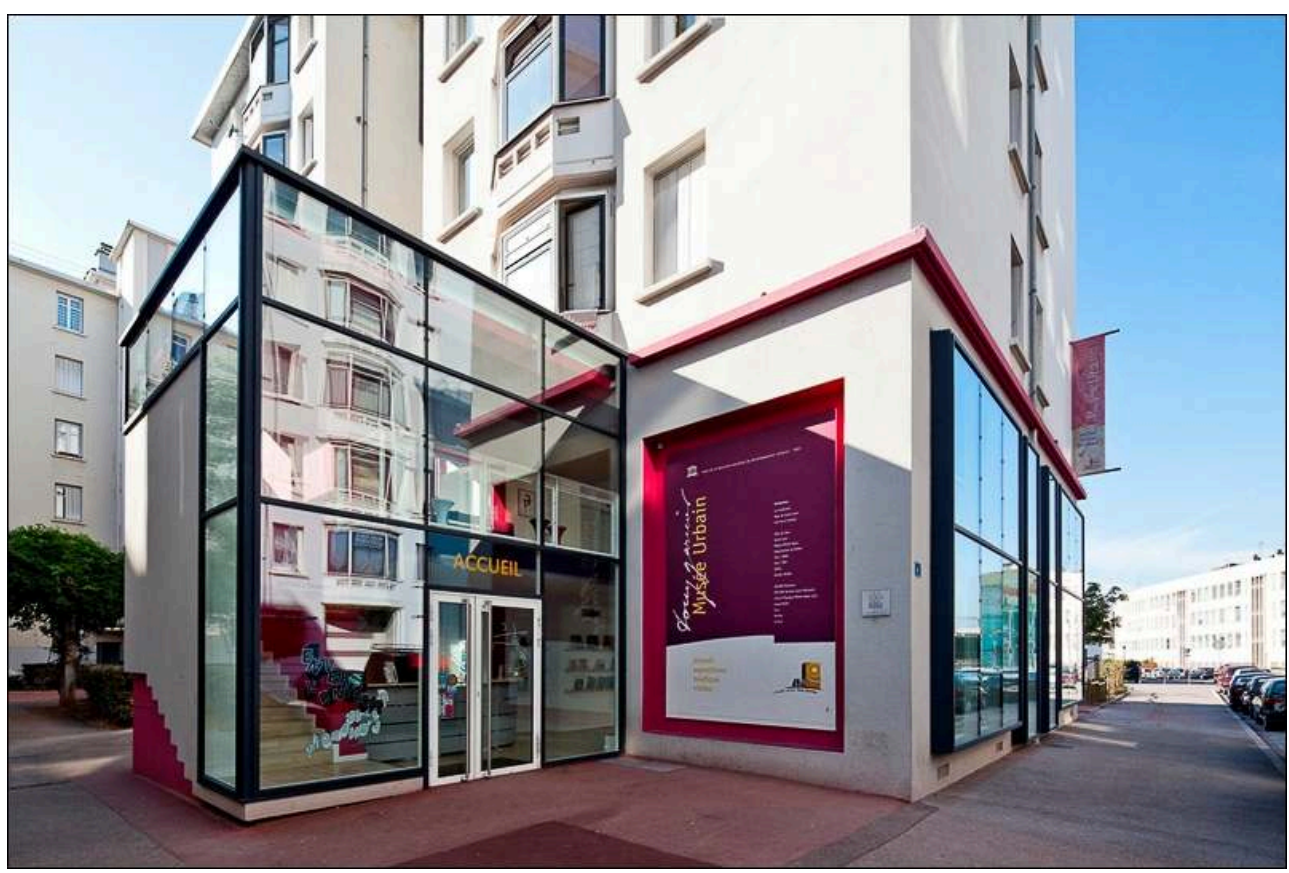

Auteur : A. Chenevez.

\section{Conclusion}

Avec l'expérience du Musée Urbain Tony Garnier, nous souhaitions montrer que si des expériences de singularisation de logements sociaux se développent, c'est précisément grâce à des arguments nouveaux liés à une forme de justice patrimoniale, avec des principes d'implication et de participation des habitants. À l'heure de la Convention pour la sauvegarde du patrimoine culturel immatériel de l'Unesco et de la Conventioncadre du Conseil de l'Europe et leurs concepts d'immatérialité, de droits culturels, de participation des habitants à la valorisation de leur cadre de vie (Gravari-Barbas, 2014), il est pertinent de nous interroger sur ce qui constitue notre bien commun et ses métamorphoses citoyennes. Le développement de nouvelles conventions internationales, telle la Déclaration de Fribourg, suscite de nouvelles légitimités patrimoniales (Lucas, 2012). Nous sortons ainsi d'un bien commun, élitiste et institutionnel pour aller vers le développement d'un patrimoine co-fabriqué par les groupes sociaux et territorialement ancré. Ces orientations sont destinées, sans nul doute, sur la longue durée, à influencer l'approche des biens communs en général et ceux des logements sociaux en particulier. Ces nouvelles conventions sont alimentées de sociologie critique et de relativisme culturel lié à l'anthropologie. Elles servent de socle et nourrissent de nouvelles causes patrimoniales. Autrement dit, il convient d'analyser en quoi les recherches critiques portées par les sciences humaines et sociales et les problématiques désormais classiques sur la gentrification produisent des effets sur le monde social et permettent des nouvelles formes de bon droit. Cela étant, force est de constater que ces arguments ne renouvellent pas toujours les acteurs de la patrimonialisation. Ses actions sont portées principalement par des classes moyennes cultivées qui en quelque sorte utilisent les arguments de justice patrimoniale pour 
s'approprier autrement et créer de nouveaux artefacts patrimoniaux. L'observation montre que ceci n'évite pas les processus de distinction culturelle et les luttes symboliques pour la reconnaissance.

\section{BIBLIOGRAPHY}

Bortolotto C., 2011. Le Patrimoine culturel immatériel. Enjeux d'une nouvelle catégorie. Paris, Éditions de la Maison des sciences de l'homme.

Becker H., 1988. Les mondes de l'art. Paris, Flammarion.

Berthet C., 1993. Des bâtisseurs aux habitants : le quartier en question. Les États-Unis à Lyon (1917-1939). In Mélanges de l'Ecole française de Rome. Italie et Méditerranée, t. 105, n² 2. p. 301-315.

Boltanski L., Thévenot L., 1991. De la justification : Les économies de la grandeur. Paris, Gallimard. Carrère G., 2012, Mémoires d'un préfet : A la traverse du XXème siècle, Paris, L'Harmattan. Coll. Graveurs de mémoire.

Chenevez A., 2012. L'expérience du Musée Urbain Tony Garnier. Documentaire audiovisuel, 14,45. Disponible en ligne : http://vimeo.com/56252190

Chenevez A., Novello Paglianti N. (Sous la direction de), 2015. L'invention de la Valeur Universelle Exceptionnelle : une utopie contemporaine. Paris, L'Harmattan, Coll. Logiques Sociales.

Davallon J., 2000. Le patrimoine : "une filiation inversée" ? Espaces Temps, Transmettre aujourd'hui. Retour vers le futur, $\mathrm{n}^{\circ} 74-75$, p. 6-16.

De Varine H., 2005. Les racines du futur, le patrimoine au service du développement local. Ed. ASdic.

Donzelot J., 2003. Faire société. La politique de la ville en France et aux États-Unis. Paris, Seuil, Coll. La couleur des idées.

Dubet F., Lapeyronnie D., 1999. Les quartiers d'exil. Paris, Seuil.

Fabre D., 2013. Le patrimoine porté par l'émotion. In D. Fabre (dir.). Émotions patrimoniales, Paris, Éditions de la Maison des Sciences de l'Homme, Coll. Ethnologie de la France, p. 13-98.

Foret C., 1992. Valorisation et dévalorisation dans la ville : le Musée Urbain ou la ré-invention d'une cité HLM. Ministère de l'équipement, Bibliothèque municipal de Lyon, exemplaire photocopié.

Gravari-Barbas M. 2014. Nouveaux défis pour le patrimoine culturel. Ateliers de réflexions prospectives (ARP), https://www.univ-paris1.fr/centres-de-recherche/eirest/projets-en-cours/ arp-nouveaux-defis-pour-le-patrimoine-culturel/

Hache E., 2011. Ce à quoi nous tenons. Propositions pour une écologie pragmatique. Paris, Éditions La Découverte, Coll. Les empêcheurs de penser en rond. 
Heinich N., 2009. La fabrique du patrimoine. De la cathédrale à la petite cuillère. Paris, Éditions de la Maison des Sciences de l'Homme, Coll. Ethnologie de la France.

Legendre P., 1985 (nouvelle édition augmentée 2004). Leçons IV. L'Inestimable Objet de la transmission. Étude sur le principe généalogique en Occident. Paris, Fayard.

Lucas J.-M. Doc Kasimir Bisou, 2012. Le PCI à l'épreuve de l'universalité. Revue Internationale de l'Imaginaire, Le patrimoine, oui, mais quel patrimoine?, n²7, Actes Sud.

Masclet O., 2005. Du "bastion" au "ghetto". Le communisme municipal en butte à l'immigration. Actes de la recherche en sciences sociales, $\mathrm{n}^{\circ} 159$, p. 10-25.

Mélé P. (sous la direction de), 2013. Conflits de proximité et dynamiques urbaines. PUF de Rennes, Coll. Géographie sociale.

Meslin K., Rousselot L., 2013. Effets sociaux des politiques urbaines de démolition et reconstruction du logement social. De l'ascension résidentielle à la fragilisation socioéconomique. Politiques sociales et familiales, vol. 114, p. 81-87.

Monnier G. (sous la direction scientifique de.), 2003. Le logement social en Seine-Saint-Denis (1850-1999). Paris, ADAGP, Editions Inventaire général, Coll. Les itinéraires du patrimoine.

Veschambre V., 2008. Traces et mémoires urbaines, enjeux sociaux de la patrimonialisation et de la démolition. Presses universitaires de Rennes.

Roz M., Lagier M., Rivet P., 1980. Tony Garnier, rapport final. Lyon, rapport dactylographié.

Saez G. et Glevarec H., 2002, Le patrimoine saisi par les associations, Ed. La Documentation Française, 2002, Coll. "Questions de culture".

Ter Minassian H., 2013. Changer Barcelone. Politiques publiques et gentrification dans le centre ancien (Ciutat Vella). Toulouse, Presses Universitaires du Mirail.

Tornatore J.-L., 2015, « Ce à quoi nous tenons et dont nous dépendons. Continuité et extériorité de l'expérience patrimoniale ", In L'invention de la Valeur Universelle Exceptionnelle : une utopie contemporaine. Paris, L'Harmattan, Coll. Logiques Sociales.

\section{NOTES}

1. "Pointe avancée de la ville de Lyon, vers le Sud-Est, la nouvelle cité des États-Unis - nullement impérialiste, nullement conquérante, comme se plaisait à le remarquer un élu socialiste - offre maintenant aux populations laborieuses un habitat parfaitement moderne et auquel il ne manque que peu de choses pour être tout à fait agréable (...) Elle s'élève fièrement sur des terrains où naguère il n'existait qu'une sorte de "zone » lépreuse. Aujourd'hui, baraquements et taudis ont disparu. Le groupe qui les a remplacés, se présente clair, net, aéré, riant. » Extrait du discours d'Edouard Herriot lors de l'inauguration de la cité Tony Garnier le 25 juin 1934. Tiré d'un article du Progrès daté du 26 juin 1934.

2. Il faut attendre l'année 1990 pour que du 7 mars au 21 mai le Centre de création industrielle (CCI) présente au Centre Pompidou à Paris une exposition sur Tony Garnier. L'objectif de cet évènement est de réhabiliter un architecte jusqu'alors peu considéré au regard de son influence considérable dans l'architecture et l'urbanisme moderne. Voir: https:// www.centrepompidou.fr/cpv/resource/cMnz64/r4reoXq

3. En 1987, le Préfet de la Région Rhône-Alpes nouvellement nommé, Gilbert Carrère, très sensible à la requalification des banlieues préconise d'installer la Direction des Affaires culturelles à Vénissieux pour modifier l'image d'un quartier dont la réputation se dégrade avec les émeutes des Minguettes. La levée des boucliers des agents de l'État empêche ce projet 
d'aboutir et la Drac s'installe alors dans un superbe bâtiment sur les quais de Saône très proche du centre historique de Lyon. (Tiré de : Les mémoires d'un préfet, Gilbert Carrère).

4. Catherine Panassier, 2009. La politique de la ville dans les années 1990 : une politique de discrimination positive pour des quartiers et des populations exclus de la ville et de la vie sociale et économique. Disponible en ligne: http://www.millenaire3.com/fileadmin/user_upload/ syntheses/Poli_Ville_Ans_90.pdf

5. La réponse des sociologues à l'appel d'offre sur «Les morphologies sociales et urbaines » en 1989 démontre l'intérêt de financer une recherche sur le Musée Urbain Tony Garnier naissant : " la Cité des « États-Unis » mérite d'être mise en avant en tant qu'élément du patrimoine de la ville de Lyon. Non pas pour ses seules qualités architecturales mais bien pour la valeur sociale et le sens éthique que lui conferent les multiples histoires anonymes qui se sont entrecroisées en ce lieu » (Voir programme de recherches: De l'ordinaire au monumental: Valeur d'usage et valorisation symbolique dans l'espace de l'habitat social. Archives privées.)

6. L'engagement est aussi facteur de mobilité sociale. Ainsi Eddie Gilles Di Pierno une des chevilles ouvrières du Comité d'intérêt local et du Musée Urbain, est aujourd'hui président de patrimoine rhônalpin et membre de la commission régionale des Monuments et des Sites.

7. Décennie mondiale du développement culturel/programme non permanent de l'UNESCO http://www.unesco.tg.refer.org/les-programmes/programme-non-permanent/decenniemondiale-du-developpement-culturel

8. L'Unesco s'oriente vers une redéfinition de la notion de patrimoine en aboutissant à l'émergence de critères plus relativistes et participatifs avec la Déclaration de Nara en 1994.

9. Eddie Gilles Di Pierno, président de patrimoine rhônalpin, administrateur de l'Union des Comités d'intérêts locaux, entretien du 26/08/2008, disponible en ligne: http:// www.millenaire3.com/Quand-la-crise-des-BANLIEUES-suscite-conscience-d.861.0.html 10. Le Progrès, 15 février 1993. Musée Urbain : Le monde au pied des murs.

\section{ABSTRACTS}

In this article, it is argued that 'heritage' is a product of history that requires cultural and political recognition if that status is to be attributed. The Etats-Unis neighbourhood of residential blocks was built in suburban Lyon during the 1930s, following the designs of Tony Garnier. The aim was to provide good housing and services for members of the working class, although some professionals also moved in. By the 1960s, many buildings had deteriorated; not until the late 1980s was restoration started. Now, the area forms the 'Tony Garnier Urban Museum', with UNESCO recognition. The transformation from dilapidated housing estate to heritage feature, with remarkable external murals, required complex negotiation between residents, planners, architects and local politicians, often involving significant social contestation.

INDEX

Subjects: Sur le Champ - Sur le Terrain 


\section{AUTHOR}

\section{ALAIN CHENEVEZ}

Alain Chenevez, alainchenevez@gmail.com, est Maître de conférences à l'Université de Bourgogne et membre du laboratoire CIMEOS (EA 4177, Dijon), Président de SEA Europe (http:// www.seaeurope.org/), il a été directeur du Musée Urbain Tony Garnier de 2004 à 2009. 